\title{
Revista Colombiana de

\section{Capítulo 2. Utilidad del mapeo tridimensional en pacientes con taquicardia nodal y taquicardias mediadas por vías anómalas}

\section{Chapter 2. Usefulness of three-dimensional mapping in patients with nodal tachycardia and anomalous pathway tachycardias}

\section{Alexander Álvarez}

Instituto del Corazón de Bucaramanga, Bucaramanga, Colombia

Recibido el 16 de noviembre de 2015; aceptado el 18 de enero de 2016

\section{Introducción}

La taquicardia por reentrada nodal constituye la taquicardia supraventricular de intervalo RR regular más frecuente ${ }^{1}, y$ comúnmente se manifiesta en forma típica y atípica. La ablación por radiofrecuencia dirigida a la vía lenta del nodo aurículo-ventricular (AV) se considera la técnica de elección en el manejo del paciente con taquicardia por reentrada nodal. Por tradición, se han utilizado dos aproximaciones, una anatómica y otra guiada por electrogramas. Aunque inicialmente se consideraron semejantes ${ }^{2,3}$, con la técnica de electrogramas se utilizaban diferentes criterios entre los autores y posteriormente se contempló que eran poco específicos del triángulo de Koch porque se encontraban también en sitios lejanos a la localización de la vía lenta. Por ello, en la actualidad la mayoría de electrofisiólogos prefieren la aproximación anatómica al asumir que la vía lenta se localiza en la porción inferior del triángulo de Koch entre el ostium del seno coronario y el anillo tricuspídeo. El éxito de esta técnica de ablación es del $95 \%$, con 2 a $5 \%$ de recurrencias, y el riesgo de bloqueo aurículo-ventricular del 0,2 al 0,6\%4.

El síndrome de Wolff-Parkinson-White (WPW) se caracteriza por la presencia de una vía anómala que se extiende a través del surco $\mathrm{AV}$; se conecta vía eléctrica desde una aurícula a un ventrículo, con conducción anterógrada (de la aurícula al ventrículo) y se asocia a síntomas como palpitaciones, síncope o muerte súbita. Esta entidad fue la primera condición curada de manera exitosa con ablación por catéter $^{5}$. El éxito del procedimiento en el síndrome de WPW es cercano al $95-98 \%^{6}$, pues en la literatura se reporta baja frecuencia de complicaciones $(<1 \%)$, muertes o accidentes cerebrovasculares en la mayoría de las series ${ }^{7}$.

\section{Utilidad del mapeo tridimensional}

Las taquicardias por reentrada nodal AV han sido foco de extensas investigaciones con delineamiento del triángulo ana- 
tómico de Koch y sus variaciones, que desempeñan un papel trascendental en la fisiología de esta arritmia. Cooke y Wilber $^{8}$ describieron su experiencia inicial en 14 pacientes con una aproximación anatómica utilizando el sistema CARTO Biosense ${ }^{\circledR}$ para crear un mapa electroanatómico de alta densidad del triángulo de Koch en ritmo sinusal y facilitar así la modificación de la vía lenta. En este estudio, el mapeo electroanatómico del triángulo de Koch podría efectuarse en menos de 30 minutos, y demarcarse áreas anatómicas como ostium del seno coronario, anillo tricuspídeo y región del haz de His. Mediante esta técnica, los autores en mención lograron limitar la liberación de energía de radiofrecuencia a un nivel por debajo de $1 \mathrm{~cm}$ de la región del His, en teoría, al reducir la posibilidad de daño del nodo $\mathrm{AV}$ y bloqueo cardiaco completo. En este estudio, se requirió una media de dos pulsos de energía de radiofrecuencia (RF) para eliminar la taquicardia por reentrada nodal sin casos de bloqueo cardiaco (fig. 1).

Así mismo, el mapeo electroanatómico del triángulo de Koch puede facilitar el entendimiento de la anatomía postquirúrgica, la cardiopatía congénita y las aurículas derechas dilatadas, en los que la distancia y orientación entre el ostium del seno coronario y el His pueden estar alteradas. Recientemente Khairy et al. ${ }^{9}$ describieron el caso de un paciente con un defecto del canal AV parcialmente reparado mediante cirugía, en el que la modificación exitosa de la vía lenta se realizó con la asistencia del sistema EnSite NavX ${ }^{\circledR}$ (St. Jude Medical).

En la figura 2 se aprecia un mapa electroanatómico de la aurícula derecha (sistema EnSite NavX) durante una taquicardia por reentrada del nodo $\mathrm{AV}$, con una activación retrógrada auricular más temprana a nivel del His. El mapeo electroanatómico tridimensional del triángulo de Koch facilitó la delineación de las entradas invertidas del nodo AV con ablación exitosa de la rama superior de la vía lenta del haz de His. Además, uno de los beneficios de utilizar mapeo electroanatómico para la taquicardia por reentrada nodal, incluye reducción del tiempo de fluoroscopia, mediante el sistema

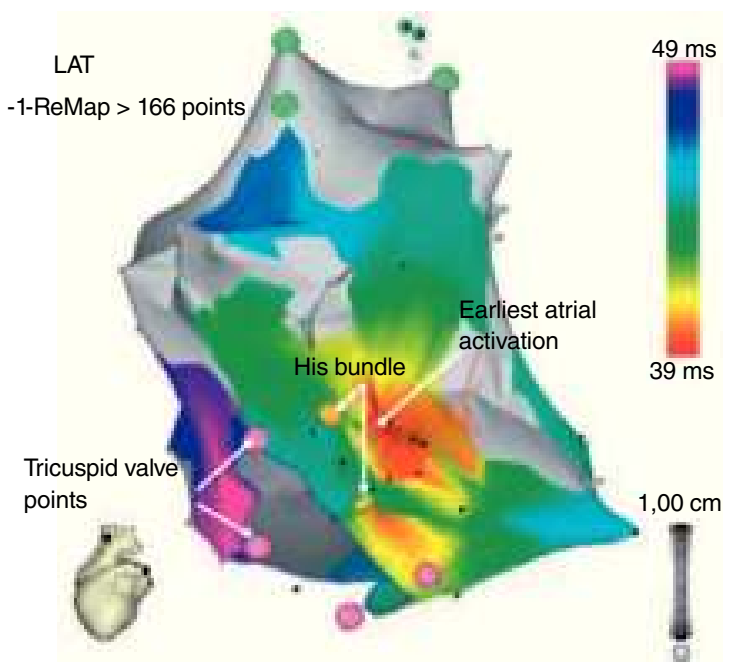

Figura 1 Mapa de activación durante taquicardia demostrada por activación auricular más temprana (área de color rojo) en la región de la vía rápida. El resto de aurícula es activada en forma concéntrica.

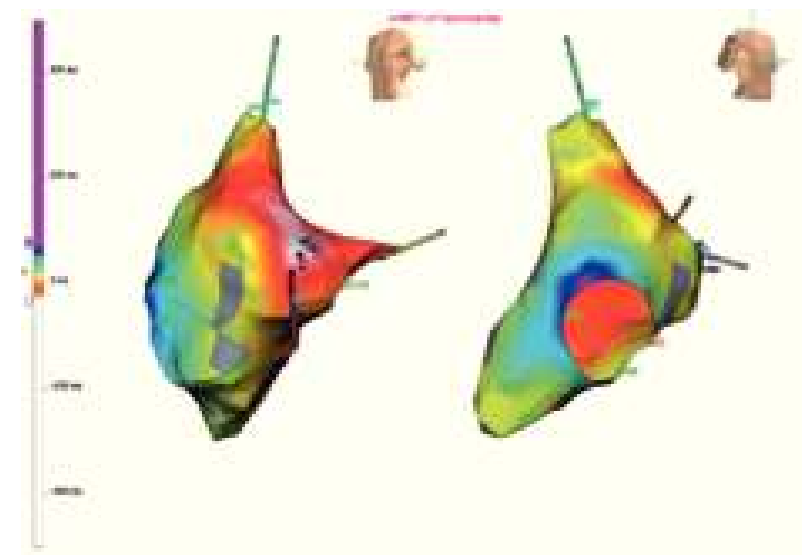

Figura 2 Mapeo electroanatómico tridimensional de activación durante una taquicardia por reentrada nodal AV con sistema EnSite NavX. Nótese la activación auricular retrógrada más temprana en el haz de His.

CARTO Biosense, en comparación con la fluoroscopia tradicional ${ }^{10,11}$. De otro lado, Kopelman et al. ${ }^{11}$ realizaron un estudio prospectivo con distribución aleatoria en el que incluyeron 20 pacientes consecutivos y compararon la modificación de la vía lenta por fluoroscopia convencional con la aproximación guiada por mapeo electroanatómico mediante el sistema CARTO Biosense, con el objetivo de evaluar el éxito del procedimiento temprano y la liberación total de energía. El éxito del procedimiento temprano fue del $100 \%$ en ambos grupos. Sin embargo, la exposición fluoroscópica y el tiempo de procedimiento fueron reducidos en el grupo del mapeo electroanatómico con un promedio de 2,7 pulsos de energía en comparación con 5 pulsos en el brazo de fluoroscopia convencional.

La utilidad de los sistemas de mapeo electroanatómico tridimensional para la localización de la inserción de las vías accesorias en la ablación con catéter ha sido investigada en estudios limitados ${ }^{10,12-15}$. Los beneficios de los sistemas de mapeo incluyeron la capacidad para delimitar los sitios de interés de difícil acceso o localización (como las vías accesorias con fisiología tipo Mahaim), navegar en áreas previamente investigadas y definir la extensión del nodo AV al reducir la probabilidad de lesión en el caso de las vías accesorias parahisianas. Adicionalmente, algunos estudios sugieren una reducción del tiempo total de fluoroscopia con el uso de sistemas de mapeo electroanatómico ${ }^{12,14,16}$ (fig. 3).

Los sistemas de mapeo electroanatómico también pueden ser útiles en casos de ablación fallida previa. En un estudio de González-Torrecilla et al. ${ }^{15}, 17$ pacientes con vías accesorias en quienes se había fallado un promedio de dos ablaciones previas con procedimientos convencionales, fueron llevados a ablación guiada por mapeo electroanatómico tridimensional (sistema CARTO Biosense) con una tasa de éxito del $94 \%$ (un caso fallido), sin recurrencia, durante un seguimiento promedio de $16 \pm 15$ meses. De igual forma, se han empleado los sistemas de mapeo electroanatómico EnSite para pacientes con ablación previa fallida de vías accesorias derechas e izquierdas y en casos de inestabilidad hemodinámica durante la arritmia (fig. 4).

De otra parte, se han descrito ablaciones exitosas de vías accesorias complejas o que representan un reto, como las 


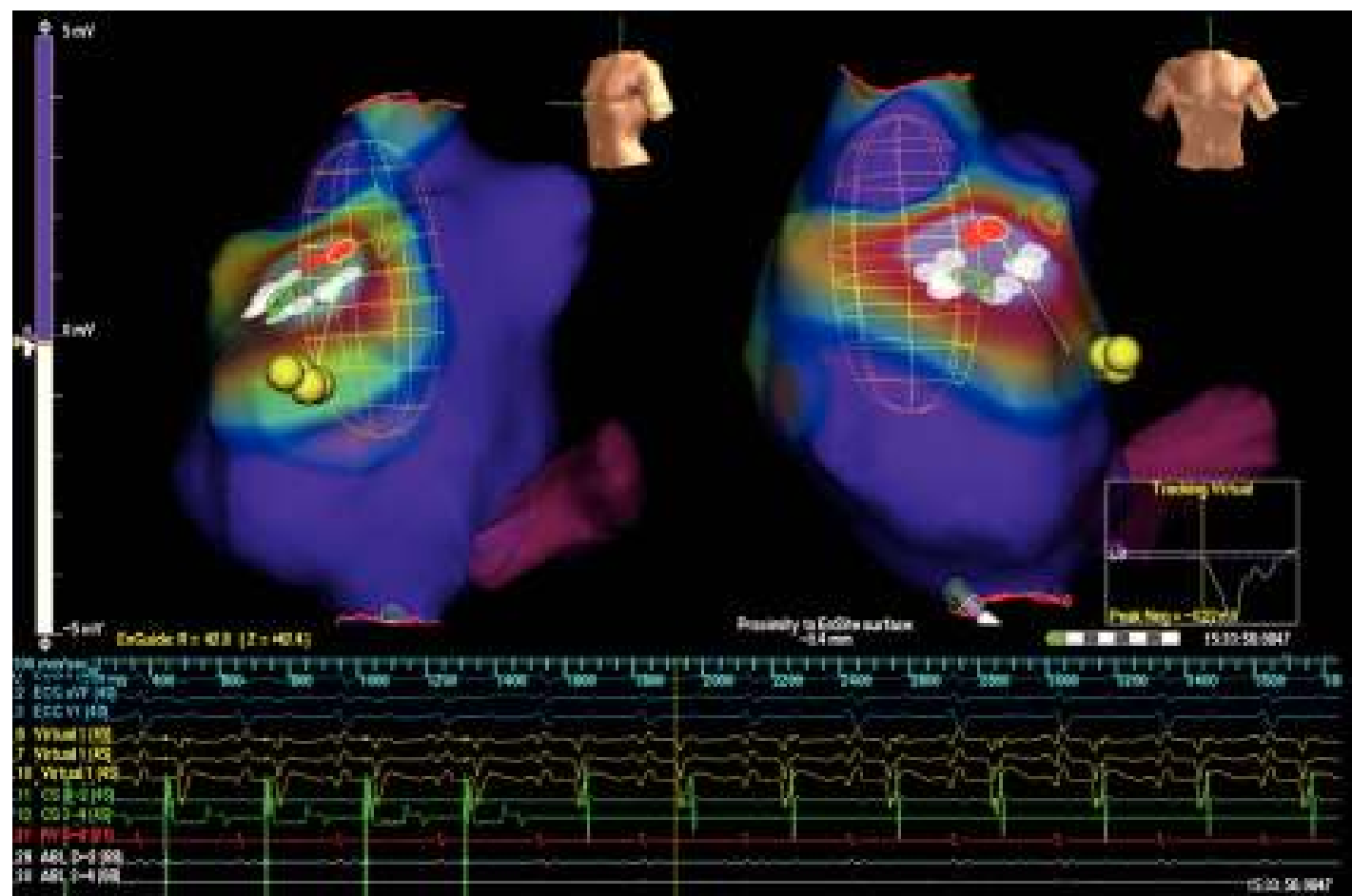

Figura 3 Mapa de activación, voltaje y propagación durante taquicardia reciprocante AV secundaria a vía accesoria derecha parahisiana con sistema electroanatómico tridimensional EnSite Array. Nótese la región del His (puntos amarillos), el seno coronario (fucsia) y las aplicaciones con energía de RF (puntos blancos) en ritmo sinusal.

asociadas con divertículos del seno coronario o anomalía de Ebstein, con la asistencia de sistemas de mapeo electroanatómico tridimensional ${ }^{17-19}$. El mapeo 3-D también se ha usado en pacientes con síndrome de WPW, con el objetivo de ma- pear vías accesorias izquierdas de localizaciones inusuales facilitando su ablación, como ocurre en el caso de las vías accesorias localizadas en el seno coronario. El mapeo 3-D ofrece la posibilidad de detallar la anatomía del seno corona-

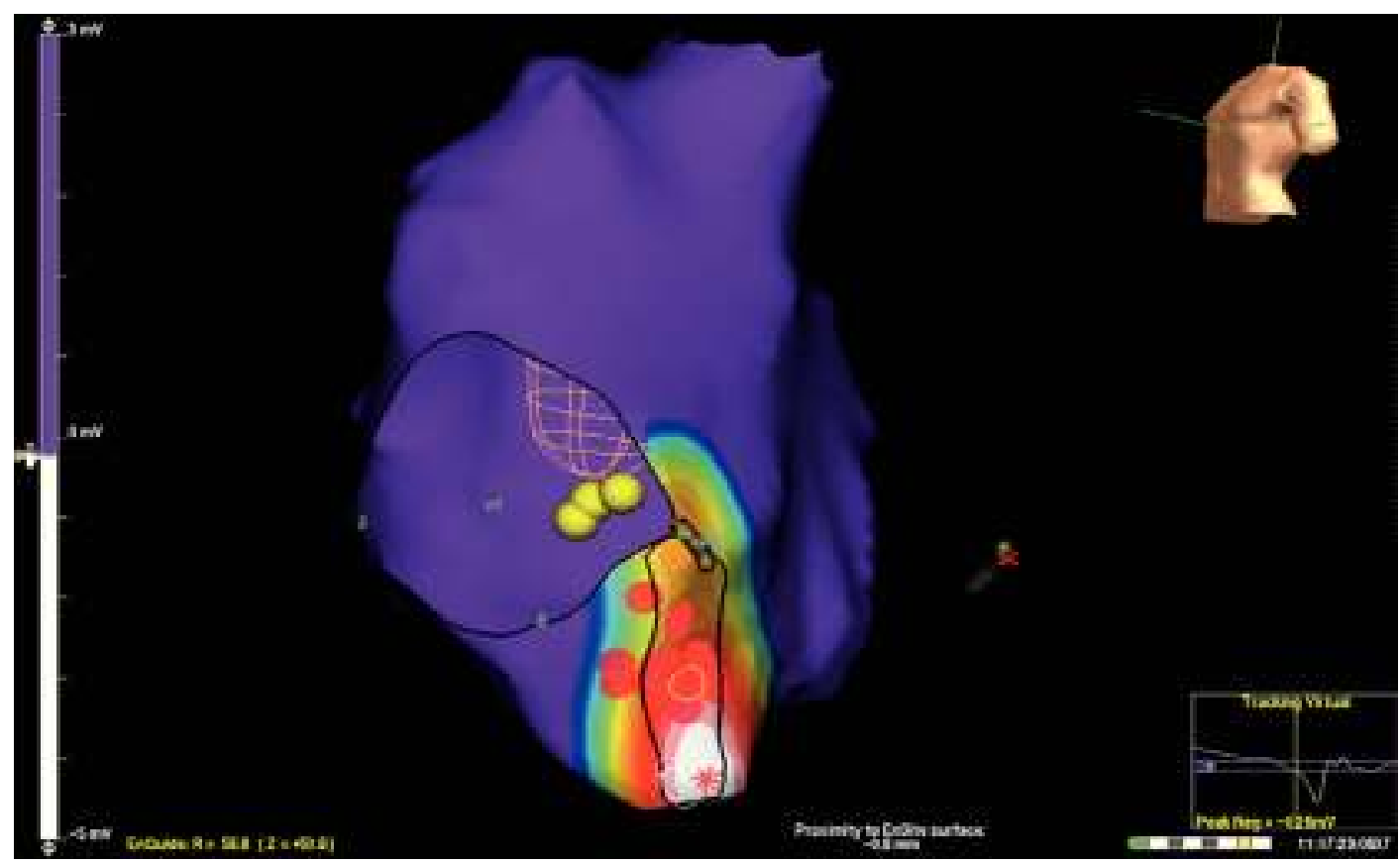

Figura 4 Mapeo electroanatómico tridimensional con mapas de activación y voltaje con sistema EnSite Balón Array, de taquicardia reciprocante AV por vía accesoria posteroseptal derecha en un paciente con ablación fallida e inestabilidad hemodinámica. Nótese el sitio de activación temprana (zona blanca) y los puntos de aplicación de RF (rojos). 
rio, las ramas, posibles aneurismas y otras alteraciones a lo largo de su curso, que pueden incluso complementarse con imágenes obtenidas mediante tomografía axial computarizada (TAC) y ser importadas al sistema de mapeo. Permite una mejor y más efectiva ablación de vías oblicuas ya que define el sitio de inserción auricular y ventricular que, dado el recorrido de estas vías, puede hacer más complejo un procedimiento de ablación convencional. En las vías parahisianas la discriminación espacial entre el sitio de inserción de la vía y el registro del His, es de importancia cardinal ya que el posicionamiento inadecuado puede llevar a bloqueo AV o fracaso del procedimiento. Por tanto, el diagnóstico preciso con mapeo 3-D y la ablación con tecnología de catéteres de contacto pueden marcar la diferencia $18,20-24$.

Para pacientes más vulnerables a los efectos deletéreos de los rayos $\mathrm{X}$, tales como mujeres embarazadas y aquellos con disfunción inmune, al igual que para casos complejos seleccionados, los procedimientos electrofisiológicos guiados con mapeo 3-D asociados a guía ecocardiográfica intracardiaca o transesofágica pueden ser una alternativa para un abordaje seguro; por ejemplo, 9 pacientes embarazadas con taquicardias supraventriculares que no mejoraron con medicación, fueron sometidas a mapeo 3-D con mínimo tiempo de fluoroscopia y sin recurrencias 25 .

En pacientes pediátricos llevados a ablación de vías accesorias, múltiples publicaciones confirman la seguridad y la eficacia del uso de un sistema de mapeo 3-D y sus beneficios, dados por la necesidad de un mínimo tiempo o no requerimiento de fluoroscopia. Además, el mapeo 3-D y la navegación no fluoroscópica han ayudado a mejorar los resultados en casos congénitos complejos y en problemas o situaciones difíciles encontradas durante el mapeo y la ablación de arritmias pediátricas; otro aspecto de importancia es la exposición considerable a radiación durante dichos procedimientos, tanto para el paciente como para los profesionales y el personal de sala ${ }^{26}$.

Las preexcitaciones ventriculares y las taquicardias por reentrada AV se observan en 20 a $30 \%$ de los pacientes con anomalía de Ebstein. Las vías accesorias se localizan alrededor del anillo tricuspídeo, incluyendo las más raras, como las fibras del tipo Mahaim. Esta anomalía se asocia adicionalmente con la presencia de múltiples vías accesorias. En los pacientes con transposición de los grandes vasos corregida, las vías accesorias se hallan en un 2 a $5 \%$ de los casos y se localizan a lo largo del lado izquierdo, en el anillo valvular AV que corresponde a la válvula tricuspídea anatómica. Durante el procedimiento de mapeo 3D y ablación el seno coronario es útil como referencia anatómica para la orientación de la válvula AV del lado izquierdo (tricúspide). En un estudio de 83 pacientes, incluyendo 17 con ventrículo único, se reportó una tasa de éxito del $82 \%$ para vías accesorias izquierdas y del 70\% para las derechas con el uso de mapeo 3-D y crioablación; en el subgrupo de pacientes con anomalía de Ebstein la tasa de éxito fue del 76 al $83 \%$, levemente menor que en los pacientes con anatomía normal cardiaca. En aquellos con múltiples vías y anatomía más compleja, la tasa de recurrencia fue mayor al $25 \%$, en especial en los niños más pequeños.

La ablación puede ser más difícil y menos exitosa a pesar del mapeo tridimensional, en pacientes con cirugías como Fontan o reemplazo valvular $\mathrm{AV}$, de ahí que deba considerarse la ablación antes de la intervención incluso cuando el paciente está asintomático ${ }^{26-29}$.
En conclusión, los sistemas de mapeo electroanatómico tridimensional han facilitado el entendimiento de los mecanismos que intervienen en las arritmias complejas y han demostrado que pueden reducir los tiempos de fluoroscopia y del procedimiento, así como mejorar el éxito de la ablación en procedimientos que habitualmente se hacen de manera convencional. La creación de lesiones de ablación lineales y la posibilidad para regresar el catéter a los sitios de ablación previos, constituyen ventajas de estos sistemas.

\section{Recomendaciones}

\section{Clase I}

- La ablación de taquicardias nodales o vías anómalas convencionales guiada por mapeo tridimensional, está indicada cuando se requiere disminuir o evitar el efecto de los rayos $\mathrm{X}$ (embarazadas y niños) (nivel de evidencia $\mathrm{C}$ ).

\section{Clase IIA}

- La ablación de taquicardias nodales o vías anómalas convencionales guiada por mapeo tridimensional, puede estar indicada cuando hay recurrencia de una ablación previa (nivel de evidencia C).

- La ablación de taquicardias nodales o vías anómalas convencionales guiada por mapeo tridimensional, puede ser útil en pacientes con anatomía cardiaca compleja (por ejemplo: anomalía de Ebstein, cardiopatía congénita, cardiopatías estructurales y posoperatorio de cirugía cardiaca) (nivel de evidencia C).

\section{Bibliografía}

1. Kwaku KF, Josephson ME. Typical AVNRT-an update on mechanisms and therapy. Cardiol Electrophysiol Rev. 2002;6:414-21.

2. Jackman WM, Beckman KJ, McClelland JH, Wang X, Friday KJ, Roman CA, et al. Treatment of supraventricular tachycardia due to atrioventricular nodal reentry, by radiofrequency catheter ablation of slow-pathway conduction. N Engl J Med. 1992;327:313-8.

3. Haissaguerre M, Gaita F, Fischer B. Elimination of atrioventricular nodal reentrant tachycardia using discrete slow potentials to guide application of radiofrequency energy. Circulation. 1992;85:2162-75.

4. Feldman A, Voskoboinik A, Kumar S, Spence S, Morton JB, Kistler PM, et al. Predictors of acute and long-term success of slow pathway ablation for atrioventricular nodal reentrant tachycardia: a single center series of 1,419 consecutive patients. Pacing Clin Electrophysiol. 2011;34:927-33.

5. Jackman WM, Wang XZ, Friday KJ, Roman CA, Moulton KP, Beckman KJ, et al. Catheter ablation of accessory atrioventricular pathways (Wolff-Parkinson-White syndrome) by radiofrequency current. N Engl J Med. 1991;324:1605-11.

6. Nakagawa H, Jackman WM. Catheter ablation of supraventricular tachycardia. Circulation. 2007;116:2465-78.

7. Bohnen M, Stevenson WG, Tedrow UB. Incidence and predictors of major complications from contemporary catheter ablation to treat cardiac arrhythmias. Heart Rhythm. 2011;11:1661-6.

8. Cooke PA, Wilber DJ. Radiofrequency catheter ablation of atrioventricular nodal reentry tachycardia utilizing nonfluoroscopic electroanatomical mapping. PACE. 1998;21:1802-9. 
9. Khairy P, Mercier LA, Dore A, Dubuc M. Partial atrioventricular canal defect with inverted atrioventricular nodal input into an inferiorly displaced atrioventricular node. Heart Rhythm. 2007; $4: 355-8$.

10. Khongphatthanayothin A, Kosar E, Nademanee E. Nonfluoroscopic three-dimensional mapping for arrhythmia ablation tool or toy? J Cardiovasc Electrophysiol. 2000;11:239-43.

11. Kopelman HA, Prater SP, Tondato F, Chronos NA, Peters N. Slow pathway catheter ablation of atrioventricular nodal re-entrant tachycardia guided by electroanatomical mapping: a randomized comparison to the conventional approach. Europace. 2003;5:171-4.

12. Sporton SC, Earley MJ, Nathan AW, Schilling RJ. Electroanatomic versus fluoroscopic mapping for catheter ablation procedures: a prospective randomized study. J Cardiovasc Electrophysiol. 2004;15:310-15.

13. Worley SJ. Use of real-time three-dimensional magnetic navigation system for radiofrequency ablation of accessory pathways. PACE. 1998;21:1636-45.

14. Drago F, Silvetti MS, Di Pino A, Grutter A, Bevilacqua M, Leibovich S. Exclusion of fluoroscopy during ablation treatment of right accessory pathway in children. J Cardiovasc Electrophysiol. 2002;13:778-82.

15. González-Torrecilla E, Arenal A, Atienza F, Almendral J. Utility of nonfluoroscopic three-dimensional electroanatomical mapping in accessory pathways with prior unsuccessful ablation techniques. Am J Cardiol. 2005;96:564-9.

16. Paydak H, Piros P, Scheinman M, Dorostkar P. Localization and radiofrequency ablation of atriofascicular pathways using electroanatomic mapping. J Electrocard. 2003;36:105-10.

17. Ai T, Ikeguchi $S$, Watanuki $M$, et al. Successful radiofrequency current catheter ablation of accessory atrioventricular pathway in Ebstein's anomaly using electroanatomic mapping. PACE. 2002;25:374-5.

18. Boulos M, Gepstein L. Electroanatomical mapping and radiofrequency ablation of an accessory pathway associated with a large aneurysm of the coronary sinus. Europace. 2004;6:608-12.
19. Eckardt L, Monnig G, Wasmer K, Breithardt G. A NavX guided cryoablation of an accessory pathway in a large coronary sinus diverticulum. J Cardiovasc Electrophysiol. 2005;16:233-4.

20. Gulletta S, Tsiachris D, Della Bella P. Catheter ablation of an anteroseptal accessory pathway guided by contact force monitoring technology and precise electroanatomical mapping. Europace. 2014;16:825-9.

21. Demazumder D, Barcelon B, Cockrell J, et al. Ablation of an anteroseptal accessory pathway from the aortic root using electroanatomic mapping. Heart Rhythm. 2014;11:2122-3.

22. Cipoletta L, Acosta J, Mont L, et al. Posterior coronary vein as the substrate for an epicardial accessory pathway. Indian Pacing Electrophysiol J. 2013;13:142-7.

23. Tan S, Sibley J, Harris S. The use of electroanatomical mapping to facilitate redo ablation of an accessory pathway. Case report. Br Heart Rhythm Soc. 2013;2:1-5.

24. Pires L, Luz Leiria T, Kruse $M$, et al. Catheter ablation of arrhythmias exclusively using electroanatomic mapping: a series of cases. Arq Bras Cardiol. 2013;101:226-32.

25. Casella M, Bartoletti S, Dello Russo A, Tondo C. 9 pregnant women with drug-refractory supraventricular tachyarrhythmias. Catheter ablation during pregnancy. J Cardiovasc Electrophysiol. 2010;21:E80.

26. McDaniel $\mathrm{G}$. The use of electroanatomic mapping systems to reduce fluoroscopic exposure in pediatric ablations. J Innovations Cardiac Rhythm Management. 2011;2:551-6.

27. Tuzcu V. A nonfluoroscopic approach for electrophysiology and catheter ablation procedures using a three-dimensional navigation system. PACE 2007;30:519-25.

28. Arroyave JA, Aristizábal JM, Velásquez J, et al. Ablación de taquicardia por reentrada intraauricular en paciente con circulación de Fontan. Disponible en: http://dx.doi.org/10.1016/j. rccar [Acceso 06 Feb 2015].

29. Aristizábal J, Uribe W, Duque M, et al. Taquicardia por reentrada nodal recurrente en paciente con transposición congénita corregida de grandes vasos. Utilización de mapeo no fluoroscópico. Arch Cardiol Mex. 2013;83:185-8. 